\title{
Validez inicial de una escala de medición del capital intelectual en universidades*
}

\section{Validity of a Scale Measuring Intellectual Capital in Universities}

Recibido: 12 de noviembre de 2014 | Aceptado: 09 de febrero de 2016

\author{
Patricia Mercado-Salgado ** \\ Universidad Autónoma del Estado de México, México \\ Pedro Gil-Monte \\ Universidad de Valencia, España \\ Daniel Arturo Cernas-Ortiz \\ Universidad Autónoma del Estado de México, México
}

doi:10.11144/Javeriana.upsy15-2.viem

Para citar este artículo: Mercado-Salgado, P., GilMonte, P., \& Cernas Ortiz, D. (2016). Validity of a Scale Measuring Intellectual Capital in Universities. Universitas Psychologica, 15(2), 109-120. http:// dx.doi.org/10.11144/Javeriana.upsy15-2. viem

Tipología de artículo. Indicar si este tuvo financiación o no por parte de alguna entidad.

** Facultad de Contaduría y Administración. Profesora de tiempo completo de la Coordinación de Investigación y Estudios Avanzados de la Facultad de Contaduría y Administración, Universidad Autónoma del Estado de México. Docente en investigación, capital humano y salud en el trabajo a nivel pregrado y posgrado. Línea de investigación: gestión del capital intelectual. Correo electrónico: pat_mersal@yahoo.com

\section{RES UMEN}

Problema: la dificultad para medir el capital intelectual en universidades latinoamericanas acentúa su poco interés, a pesar de su relevancia en la economía del conocimiento. Objetivo: construir y validar una escala de medición del capital intelectual de universidades a partir de la teoría de recursos y capacidades y un modelo de tres componentes (capital humano, capital estructural y capital relacional). Método: esta escala fue elaborada por un grupo de expertos utilizando análisis de contenido. La prueba empírica se hizo con una muestra de conveniencia $(n=742)$ de académicos, mandos medios y directivos de universidades mexicanas. Resultados y conclusiones: con el análisis factorial exploratorio se detectaron cuatro factores (capital humano, capital estructural, capital relacional y capital tecnológico) que explican el 66.74\% de la varianza. El Alpha de Crobach fue mayor a 0.85. Se concluyen avances en la calidad métrica y estabilidad, pero queda en el tintero replicar el estudio para generar modelos de medida y estructural.

Palabras clave

capital intelectual; escala de medición; universidades. Código JEL: I2, M1

\section{A B S T R A C T}

Problem: The difficulty to measure the IC in Latin American universities highlights its little interest even though its relevance to the knowledge economy. Purpose: Design and validate a survey for measure Intellectual Capital in universities from a Resource-based Theory perspective and a three components model (human capital, structural capital and relational capital). Method: A subject matter expert panel technique was used to generate the items. It was empirically tested with a convenience sample $(n=742)$ of academics, middle managers and principals of institutions of higher education in México. Results and Conclusions: With exploratory factor analysis and principal components analysis methods, four factors were detected (human capital, structural capital, relational capital and technological capital) explaining total variance of $66.74 \%$. All subscales obtained Cronbach's Alpha higher to 0.80 . There were advances on the measure quality and stability of the questionnaire. In order to generate measure and structural models this study could be replicated.

Keywords

Intelectual Capital; Measure Scale; University. JEL Classification: I2, M1 


\section{Introducción}

Las universidades desempeñan un papel clave en el crecimiento económico y la competitividad global. Al mismo tiempo, viven en una carrera para llegar a ser de rango mundial. Lograrlo requiere tomar en cuenta dos perspectivas complementarias: una de carácter externo y otra de carácter interno (Salmi, 2009). La primera se refiere a la función y los recursos del gobierno nacional, estatal y local. La segunda son los pasos a seguir para que cada institución busque su transformación, lo que requiere de un fuerte liderazgo, una visión audaz de la misión, objetivos y planes estratégicos articulados, así como el compromiso de evaluar de manera objetiva sus puntos fuertes y las áreas que necesitan mejorar para apoyar prácticas de gestión, incluyendo sistemas de control, indicadores de resultados y mecanismos de rendición de cuentas.

Kaplan y Norton (1992) reconocen que las medidas financieras ya no son suficientes para generar comparaciones; también son necesarias medidas no financieras, aquellas que corresponden a la inversión en intangibles como el capital intelectual.

La literatura (CIC, 2012; Lyn \& Bozeman, 2006) avala la construcción de medidas no financieras del capital intelectual, principalmente con información estadística (indicadores). Aunque con menor frecuencia también se refieren encuestas, cuestionarios y entrevistas para construir diagnósticos a partir de la percepción de quienes integran las organizaciones, lo cual no deja de criticarse por su carácter subjetivo, dinámico y hasta fugaz (Sánchez, Elena, \& Castrillo, 2009).

El objetivo de esta investigación es construir y validar una escala para la medición del capital intelectual en universidades, con la finalidad de que tanto investigadores como tomadores de decisiones cuenten con una herramienta objetiva y efectiva para continuar enriqueciendo el campo de conocimiento de los intangibles.

\section{Revisión de literatura}

El capital intelectual es un tema emergente para académicos, gobernantes e inversionistas (Bezhani, 2010) y ha sido investigado en naciones industria- lizadas, pues se reconoce como conductor del crecimiento organizacional en una economía basada en el conocimiento (Lev \& Zambon, 2003). Sin embargo, todavía son insuficientes los estudios en economías emergentes y, específicamente, en universidades (Tseng \& James Goo, 2005).

\section{El capital intelectual desde la teoría de recursos y capacidades}

Esta perspectiva, sacada a la luz por Edith Penrose como una teoría de crecimiento organizacional basada en el conocimiento (Barney, 1991), establece que la ventaja competitiva de una organización está en función del control interno y la combinación de sus recursos, lo que incluye activos, capacidades, procesos organizacionales, información y conocimiento, entre otros (Sánchez, 2006).

El capital intelectual es la combinación de recursos intangibles y capacidades valiosos que permiten a la organización transformar activos financieros, materiales y humanos en un sistema capaz de crear valor para todos los agentes vinculados a ella (Sánchez et al., 2009). Este intangible encierra la dinámica (actividades) para lograr que el conocimiento, como la combinación de recursos y capacidades, genere valor para quienes pertenecen a ella, para ella misma y para la sociedad en la que está inmersa.

Desde hace más de una década han surgido modelos de capital intelectual; ${ }^{1}$ todos comparten su carácter intangible, el valor que generan a la organización y están representados, en esencia, por tres componentes: capital humano, capital estructural y capital relacional.

El capital humano es el conocimiento tácito y explícito, la capacidad de aprender, crear y generar; es las competencias, el talento y la experiencia aplicados a soluciones efectivas y creativas. El capital estructural es el conocimiento que la organi-

1 Algunos modelos de capital intelectual son: Balance Scorecard de Kaplan y Norton (1992), Technology Brooker de Ann Brooking (1997), Navegador Skandia de Edvinsson y Malone (1997), Índice de Capital Intelectual de Roos, Roos, Dragonetti, \& Edvinson (1997), Monitor de Activos Intangibles (Sveiby, 1997), Modelo de capital intelectual de Stewart (1997), Modelo diamante de Bontis (1998) y Modelo Intellectus Euroforum (CIC, 2012). 
zación logra explicitar, sistematizar y socializar; es la estructura jerárquica, los procedimientos y las políticas; es soporte logístico y redes de apoyo. El capital relacional es el conocimiento que revisten las relaciones entre la organización y sus stakeholders (clientes, inversionistas, proveedores, agencias gubernamentales, etc.), así como la imagen que se construye de la organización hacia el exterior (Bontis, 1998; Brooking, 1997; Stewart, 1997).

\section{Medición del capital intelectual en la universidad}

Las universidades sometidas a un proceso de transformación profunda deben gestionar el capital intelectual como opción proactiva centrada en el conocimiento (Bezhani, 2010) ya que, por un lado, su misión está en formar personal calificado, generar conocimiento y difundir la cultura; por el otro, son pocas las acciones hacia la generación de recursos propios, debiendo optimizar lo que tienen disponible. Sin embargo, y teniendo en cuenta que el capital intelectual en las universidades públicas mexicanas ha sido poco explorado, todavía no se aprecia su sentido práctico: la generación de valor (Nava \& Mercado, 2010).

No medir y no informar sobre el capital intelectual desencadena daños en las universidades. Esta omisión se traduce en costos excesivos de capital y alta volatilidad, inversiones insuficientes, deficiente asignación de recursos, peligro de una incorrecta valuación, así como el desconocimiento o falta de información sobre estos intangibles para agentes internos y externos (Caredda, D'Egidio, \& Gasperini, 2004; Mouritsen, Bukh \& Marr, 2004).

Predominan dos corrientes sobre la medición del capital intelectual, una objetiva y otra subjetiva. La primera parte de la construcción de indicadores con información secundaria. Algunas propuestas generadas son (Sánchez et al., 2009): el reporte del capital intelectual - RICARDIS, a cargo de la Comisión Europea, para mejorar la investigación, el desarrollo y la innovación en pequeñas y medianas empresas y el reporte de capital intelectual en centros de investigación en Australia, el cual desde 2006 es obligatorio para las universidades.
Para la segunda corriente, el capital intelectual como constructo es una abstracción que puede medirse indirectamente y al reflejarlo en una escala se convierte en una variable latente (Martínez, Hernández, \& Hernández, 2006). Como tal, su fuerza o magnitud cambia, y esto es precisamente lo que la escala aquí propuesta pretende estimar: la magnitud de los componentes del capital intelectual para universidades, considerando para ello que no existen mediciones únicas y todos los instrumentos de medición están basados en un determinado número de dominios, es decir, un constructo no queda representado completamente en un solo cuestionario.

\section{Método}

El paradigma de esta investigación aplicada fue cuantitativo, aunque se partió de técnicas cualitativas para la elaboración de los reactivos. En primer lugar, un grupo de expertos elaboró el cuestionario utilizando análisis de contenido; en segundo lugar, y a partir de evidencia empírica (aplicación del cuestionario), se realizaron las pruebas estadísticas para detectar sus cualidades métricas.

\section{Participantes}

Se aplicó la encuesta elaborada a 750 sujetos voluntarios que se desempeñaban como directivos, mandos medios o académicos en tres instituciones de educación superior de México: una del norte, otra del centro y la tercera del sureste de México. Se decidió por este perfil de unidad de análisis por su participación e influencia en la construcción de la estrategia, el control y la retroalimentación a nivel organizacional.

De los participantes, $51.6 \%$ son hombres, $75 \%$ tiene posgrado (46\% maestría y $29 \%$ doctorado), $19.7 \%$ es personal administrativo, $52.8 \%$ es académico y $27.6 \%$ tiene la categoría académico-administrativo; por el puesto desempeñado, $48.7 \%$ es directivo y mando medio y $51.3 \%$ de los encuestados se desempeñan como académicos. Ocho de cada diez participantes tienen horas frente a grupo, impartiendo en promedio 10 horas/semana $(\square=7.08)$. 
La antigüedad en la universidad va desde menos de un año hasta 45 años $(\tilde{x} \tilde{x}=12.57, \sigma=10.19)$. La antigüedad en el puesto $(\tilde{x} \tilde{x}=7.18, \sigma=8.04)$ refleja que existe rotación interna, lo cual está vinculado a la conformación periódica de los órganos colegiados y la junta directiva.

\section{Instrumento}

La primera versión del cuestionario quedó integrada por 45 reactivos redactados en sentido afirmativo (15 para capital humano, 15 para capital estructural y 15 para capital relacional). Para llegar a esta versión se inició con la técnica traducción-retraducción de dos cuestionarios de capital intelectual en idioma inglés (Huang \& Jim Wu, 2010; Nazari, Herremans, Manassian, \& Kline, 2011). Ante su incompatibilidad con el contexto de las universidades mexicanas, se procedió a la elaboración de la escala de capital intelectual bajo la dinámica de discusión en grupo con expertos. A partir de las definiciones de capital humano, capital estructural y capital relacional de modelos de capital intelectual, se generaron los ítems mediante la técnica de análisis de contenido.

Se optó por la escala Guthman como formato de respuesta múltiple. Cada ítem tiene un par de adjetivos en los extremos: 1 (inadecuado) a 10 (adecuado), en donde bajas puntuaciones reflejan poco capital intelectual. También se incluyeron siete reactivos en escala nominal y de razón: sexo, nivel de escolaridad, puesto desempeñado, categoría de contratación, práctica de la docencia (horas frente a grupo), antigüedad en el organismo académico y antigüedad en el puesto.

\section{Procedimiento}

Aplicación del cuestionario. Inició con la solicitud a directivos de los organismos académicos de las universidades y se garantizó el anonimato a los respondientes. La recolección de datos primarios se llevó a cabo entre mayo de 2012 y diciembre de 2013. Por el periodo de aplicación, se trata de un estudio sincrónico, toda vez que el contexto no logra variaciones en periodos cortos.
Análisis de datos. Utilizando el paquete estadístico SPSS versión 20.0, se pusieron a prueba las propiedades psicométricas de la escala de capital intelectual, se examinó la consistencia interna (alpha de Cronbach) y la validez de constructo mediante análisis factorial exploratorio con el método de componentes principales y rotación Varimax. El criterio para retener los factores fue eigenvalue mayor que 1. A partir de los antecedentes científicos abordados en apartados anteriores y la significancia conceptual, se asignaron etiquetas a los factores con los ítems que tienen en él pesos mayores a 0.5, con lo cual se evitó privilegiar la técnica estadística sobre el fundamento teórico de un intangible poco conocido y aplicado en la estrategia organizacional, como el capital intelectual.

Previo al análisis estadístico, se detectaron casos atípicos (outliers) en la base de datos que pudieran afectar los resultados. Siguiendo el criterio de que aquellos valores entre 2 y 2.5 deben ser eliminados (Tarling, 2008), se quitaron ocho cuestionarios que arrojaron índices de anomalía entre 2.128 y 2.396. Por lo tanto, la base de datos sobre la que se generaron resultados fue de 742 .

\section{Resultados}

\section{Calidad de los reactivos}

Su finalidad es doble. Por un lado, eliminar cualquier reactivo que no sea útil, pues mientras más corto sea el cuestionario y represente mejor a la variable, la escala será de mayor calidad. Por el otro, independiente a la proporción de sujetos y variables, la muestra debe tener la heterogeneidad que es normal encontrar en la población de estudio (Field, 2003). Para ello, se obtuvieron medidas de tendencia central (media, desviación estándar y asimetría) y correlaciones intertest. El poder de discriminación es mejor si el promedio se encuentra cerca del punto medio de la escala (5.5) y la desviación estándar es superior a 1.5, lo que indica que una buena proporción de las personas encuestadas elige los diferentes valores de la escala. La media de los reactivos osciló entre 6.78 y 8.36; la media más baja correspondió al reactivo 32 ("la comunicación 
de los directivos, profesores y administrativos con los alumnos") y la media más alta al reactivo 45 ("la reputación del organismo académico"), ambos de la escala de capital relacional. Las desviaciones estándar estuvieron cercanas y superiores a 1.5 en todos los casos. Puede decirse que existió polarización de las respuestas del personal en relación con los contenidos de los reactivos o bien, que los ítems sí contaron con poder de discriminación.

Se recurrió a la asimetría y a su error estándar (0.09), los datos de este estudio cumplen con este criterio. Todos los reactivos presentaron un valor de asimetría dentro del intervalo $+/-2$ lo que indica que su desviación de la normalidad no es relevante (Miles \& Shevlin, 2005).

Las correlaciones intertest ayudan a comprobar que los reactivos se correlacionen bien entre sí y con la puntuación total del test ${ }^{2}$ (Clark-Carter, 2002). En este caso, todos los reactivos reflejaron su pertenencia a la escala, considerando aquellos valores estadísticamente significativos iguales o superiores a 0.5 (Morales, 2011).

\section{Análisis factorial exploratorio}

La suficiencia del tamaño de muestra cumplió con los criterios conexos sugeridos por Nunnaly (2009) en cuanto a la proporción de sujetos con respecto a las variables $(\mathrm{N}=10 \mathrm{k})$, al número de variables (mayor a 20) y a la claridad de la estructura teórica del constructo. La muestra fue de 742 sujetos, 45 variables (o reactivos del cuestionario) y la composición del capital intelectual se conformó por capital humano, capital estructural y capital relacional.

En cuanto a la pertinencia de la matriz de correlaciones, se calculó la prueba Kaiser-Meyer-Olkin $(\mathrm{KMO}=0.980)$. Al rebasar 0.5 se consideró buen criterio, ya que mientras más pequeño, mayor es el valor de los coeficientes de relación parcial y, por

2 Quienes dan una puntuación alta a una afirmación, tenderán a dar una alta a la otra, y quienes confieren una baja puntuación a una, tenderán a otorgar una baja a la otra. La afirmación que no se correlaciona razonablemente bien con la calificación total ni con otras afirmaciones debe descartarse, pues demuestra que no es una afirmación de importancia (Clark-Carter, 2002, pp. 94-95). lo tanto, menos deseable (Morales, 2011). La prueba de esfericidad de Bartlett arrojó un valor alto (Chi-cuadrado aproximado $=29141.425, \mathrm{p} \leq$ ) $)$, lo que significa que las correlaciones de la matriz son apropiadas para el análisis factorial exploratorio o que los datos se adecuan al modelo propuesto (Aron \& Aron, 2001).

Con lo anterior como condición, se calculó el análisis factorial exploratorio por el método de componentes principales y rotación Varimax para establecer la validez de constructo de la encuesta elaborada para la medición del capital intelectual.

Los resultados alcanzaron a explicar una varianza total de $65.48 \%$. Se obtuvieron cuatro y no tres factores. Los cuatro factores obtenidos estuvieron sólidamente definidos con 18, 14, 7 y 6 reactivos, con cargas factoriales mayores a 0.5 (a excepción del reactivo 29 que obtuvo 0.411). Estas cargas factoriales son ya de clara relevancia práctica y definen bien los factores (Hair, Anderson, Tatham, \& Black, 1999). La varianza que explica cada uno de los factores (después de la rotación) fue 20.89\%, $18.78 \%, 14.31 \%$ y $11.50 \%$.

Sin embargo, al revisar la pertenencia inicial de los reactivos a los tres factores ( 1 a 15 para capital humano; 16 a 30 para capital estructural; 31 a 45 para capital relacional), se detectaron las siguientes inconsistencias: (a) los ítems 10 al 15 cargaron al factor de capital estructural, siendo construidos para el de capital humano; (b) los ítems 16 al 20 generaron el cuarto factor y eran parte del capital estructural; (c) la variable 23 (sistema de control escolar) se concibió dentro del capital estructural y tuvo mayor carga en el cuarto factor $(0.534)$ que en aquel (0.381); (d) el reactivo 26 (programas educativos de licenciatura y posgrado) apareció en capital relacional cuando se originó en el estructural; y (e) el ítem 31 (comunicación entre personal y alumnos) cargó en capital estructural y se construyó para el relacional.

Los dos primeros casos pueden tratarse de oportunidades para mover la frontera del conocimiento, ya que las condiciones estadísticas por sí solas no garantizan resultados con significado conceptual; esto es, la relevancia de un factor no depende exclusivamente de las características estadísticas, sino 
TABLA 1

Matriz de componentes principales rotación Varimax ( $n=742$ ) (41 items y $66.74 \%$ varianza total explicada)

\begin{tabular}{|c|c|c|c|c|}
\hline \multirow[b]{2}{*}{ Reactivos } & \multicolumn{4}{|c|}{ Cargas factoriales } \\
\hline & $\begin{array}{c}\text { Factor } 1 \\
\text { capital } \\
\text { estructural }\end{array}$ & $\begin{array}{c}\text { Factor } 2 \\
\text { capital } \\
\text { relacional }\end{array}$ & $\begin{array}{c}\text { Factor } 3 \\
\text { capital } \\
\text { humano }\end{array}$ & $\begin{array}{c}\text { Factor } 4 \\
\text { capital } \\
\text { tecnológico }\end{array}$ \\
\hline 08. Capacitación a los empleados & 0.58 & 0.26 & 0.45 & 0.21 \\
\hline 09. Trabajo en equipo & 0.61 & 0.28 & 0.48 & 0.13 \\
\hline 10. Liderazgo & 0.69 & 0.28 & 0.36 & 0.06 \\
\hline 11. Satisfacción laboral & 0.69 & 0.23 & 0.37 & 0.14 \\
\hline 12. Motivación & 0.68 & 0.28 & 0.41 & 0.18 \\
\hline 13. Soporte socio emotivo & 0.72 & 0.28 & 0.33 & 0.19 \\
\hline 14. Relación entre los empleados & 0.56 & 0.32 & 0.48 & 0.22 \\
\hline 15. Relación entre directivos y mandos medios & 0.65 & 0.33 & 0.34 & 0.23 \\
\hline 21. Niveles jerárquicos & 0.60 & 0.36 & 0.16 & 0.43 \\
\hline 22. Puestos académicos y administrativos & 0.61 & 0.34 & 0.20 & 0.37 \\
\hline 24. Sistema de planeación y control de personal & 0.58 & 0.35 & 0.24 & 0.25 \\
\hline 25. Sistema de evaluación y promoción de personal & 0.64 & 0.35 & 0.23 & 0.28 \\
\hline 27. Manuales y procedimientos & 0.54 & 0.41 & 0.23 & 0.27 \\
\hline 28. Gestión del conocimiento & 0.56 & 0.40 & 0.18 & 0.28 \\
\hline 30. Cultura organizacional & 0.54 & 0.46 & 0.28 & 0.29 \\
\hline 32. Comunicación entre personal y padres de familia & 0.52 & 0.44 & 0.14 & 0.25 \\
\hline 33. Comunicación con empleadores & 0.45 & 0.58 & 0.14 & 0.28 \\
\hline 34. Confianza de alumnos hacia la institución & 0.46 & 0.59 & 0.21 & 0.26 \\
\hline 35. Confianza de los empleadores & 0.28 & 0.66 & 0.21 & 0.22 \\
\hline 36. Confianza de los egresados & 0.28 & 0.66 & 0.24 & 0.18 \\
\hline 37. Relación con administración central & 0.43 & 0.55 & 0.20 & 0.21 \\
\hline 38. Relación con instituciones de educación media & 0.44 & 0.58 & 0.19 & 0.15 \\
\hline 39. Relación con instituciones hermanas & 0.37 & 0.68 & 0.20 & 0.18 \\
\hline 40. Contribución a la sociedad & 0.18 & 0.72 & 0.31 & 0.16 \\
\hline 41. Vinculación a la sociedad & 0.22 & 0.69 & 0.31 & 0.20 \\
\hline 42. Extensión de los servicios universitarios & 0.25 & 0.69 & 0.28 & 0.24 \\
\hline 43. Difusión de la cultura & 0.36 & 0.59 & 0.21 & 0.27 \\
\hline 44. Responsabilidad social & 0.36 & 0.64 & 0.24 & 0.14 \\
\hline 45. Reputación del organismo académico & 0.16 & 0.68 & 0.31 & 0.22 \\
\hline 01. Nivel educativo del personal & 0.24 & 0.26 & 0.61 & 0.32 \\
\hline 02. Experiencia del personal & 0.19 & 0.29 & 0.71 & 0.24 \\
\hline 03. Creatividad de los empleados & 0.31 & 0.29 & 0.69 & 0.18 \\
\hline 04. Aptitudes y capacidades de los empleados & 0.24 & 0.27 & 0.77 & 0.16 \\
\hline 05. Resolución de problemas & 0.34 & 0.24 & 0.72 & 0.20 \\
\hline 06. Obtención de resultados & 0.42 & 0.28 & 0.65 & 0.22 \\
\hline 07. Actitud hacia el aprendizaje & 0.34 & 0.27 & 0.68 & 0.16 \\
\hline 16. Conectividad a internet & 0.13 & 0.15 & 0.25 & 0.72 \\
\hline 17. Equipo de cómputo (software y hardware) & 0.26 & 0.18 & 0.23 & 0.72 \\
\hline 18. Infraestructura asignada a profesores & 0.16 & 0.30 & 0.17 & 0.74 \\
\hline 19. Infraestructura para enseñanza-aprendizaje & 0.21 & 0.36 & 0.21 & 0.66 \\
\hline 20. Infraestructura para educación a distancia & 0.32 & 0.21 & 0.14 & 0.65 \\
\hline Porcentaje de la varianza explicada tras la rotación & $21.01 \%$ & $19.91 \%$ & $14.91 \%$ & $10.91 \%$ \\
\hline
\end{tabular}

Fuente: elaboración propia 
del contenido teórico de las variables. El siguiente apartado contiene una discusión al respecto.

La naturaleza de las irregularidades de los reactivos 23, 26 y 31 sí apuntan a su eliminación.

Se procedió al segundo cálculo del análisis factorial exploratorio por el mismo método para 42 ítems, confirmando los requisitos de pertinencia de la matriz de correlaciones $(\mathrm{KMO}=0.979$ y esfericidad de Bartlett con Chi-cuadrado aproximado $=27188.613, \mathrm{p} \leq 0)$. La varianza se incrementó a $66.24 \%$, permaneciendo cuatro factores con varianzas de $20.92 \%, 19.61 \%, 14.79 \%$ y $10.91 \%$. Sin embargo, el reactivo 29 (investigación y producción científicas) se desplazó al cuarto factor cayendo en una inconsistencia conceptual, por lo que para el tercer y último cálculo del análisis factorial exploratorio se decidió su eliminación (tabla 1). Con 41 reactivos se incrementó la varianza explicada a $66.74 \%(\mathrm{KMO}=0.979$ y esfericidad de Bartlett con Chi-cuadrado aproximado $=26514.415, \mathrm{p} \leq$ 0 ), no se perdió estabilidad en las cargas factoriales de los ítems entre el primero y el tercer cálculo del análisis factorial exploratorio y las varianzas de los cuatro factores (después de la rotación) mejoraron a 21.01\% (16 reactivos), 19.91\% (13 reactivos), 14.91\% (7 reactivos) y $10.91 \%$ (5 reactivos). En el anexo pueden verse los reactivos.

\section{Confiabilidad de los factores}

La medida resultante siempre está afectada por un error aleatorio en diversas aplicaciones, por lo que se busca una tendencia en la consistencia de un conjunto de medidas (Duane, 2008). Dicha estabilidad responde a qué tanto se pueden generalizar los resultados en muestras similares (Kerlinger $\&$ Lee, 2002). La primera prueba a calcular es el Alpha de Cronbach. Además de los descriptivos (media y desviación estándar), la tabla 2 contiene la confiabilidad de los cuatro factores referidos (en diagonal), siendo mejor para el capital estructural (0.96), seguida del capital relacional (0.95), el capital humano (0.93) y el capital tecnológico (0.86). En todos los casos, la confiabilidad es mayor a $0.70 \mathrm{y}$, de acuerdo a Nunnally (2009), los valores de 0.70 sirven para investigación exploratoria, los de 0.80 son útiles en investigación básica y los de 0.90 (o mejor) funcionan en los entornos aplicados en los que se tomarán decisiones importantes, sobre todo en las organizaciones (Duane, 2008). Estos resultados contribuyeron a confirmar la consistencia interna de la escala de medición del capital intelectual en universidades.

También se obtuvo la correlación de Pearson para explorar si las cuatro subescalas corresponden al capital intelectual, llegando a relaciones moderadamente altas y significativas, con lo que se predice una asociación entre ellas tal como se propuso en el marco de la teoría de recursos y capacidades.

El capital humano $(\bar{x}=\bar{x}=7.75, \sigma=1.36)$ tiene un sentido bidireccional con el capital estructural $(\mathrm{r}=0.801, \mathrm{p}>0.01)$ y con el capital relacional $(\mathrm{r}=0.732, \mathrm{p}>0.01)$, es decir, se detectan relaciones de asociación, pero no de causalidad. Por su parte,

Tabla 2

Correlación entre factores del CI $(n=742)$

\begin{tabular}{cccccccc}
\hline Factores & $\begin{array}{c}\text { No. de } \\
\text { reactivos }\end{array}$ & Media & DE & $\begin{array}{c}\text { Capital } \\
\text { humano }\end{array}$ & $\begin{array}{c}\text { Capital } \\
\text { estructural }\end{array}$ & $\begin{array}{c}\text { Capital } \\
\text { relacional }\end{array}$ & $\begin{array}{c}\text { Capital } \\
\text { tecnológico }\end{array}$ \\
\hline $\begin{array}{c}\text { Capital } \\
\text { humano }\end{array}$ & 7 & 7.75 & 1.36 & $(0.93)$ & $0.80^{* *}$ & $0.73^{* *}$ & $0.62^{* * *}$ \\
$\begin{array}{c}\text { Capital } \\
\text { estructural }\end{array}$ & 16 & 7.49 & 1.57 & & $(0.96)$ & $0.86^{* *}$ & $0.68^{* *}$ \\
$\begin{array}{c}\text { Capital } \\
\text { relacional } \\
\begin{array}{c}\text { Capital } \\
\text { tecnológico }\end{array}\end{array}$ & 13 & 7.89 & 1.32 & & & $(0.95)$ & $0.67^{* *}$ \\
\hline
\end{tabular}

** La correlación es significativa a 0.01 (bilateral)

Fuente: elaboración propia 
el capital estructural $(\bar{x}=\bar{x}=7.49, \sigma=1.57)$ refleja un vínculo fuerte con el capital relacional $(\mathrm{r}=0.855$, $\mathrm{p}>0.01)$. Si bien en este estudio no se identifican relaciones de causa-efecto, Bontis (1998) soporta su modelo de 'diamante' a partir de la interrelación de tres componentes del capital intelectual. Además de lo anterior, y como lo señala Sullivan (2001), el capital tecnológico $(\bar{x}=\bar{x}=7.41, \sigma=1.73)$ sirve de apoyo para el capital humano $(\mathrm{r}=0.616, \mathrm{p}>0.01)$, el capital estructural $(0.683, \mathrm{p}>0.01)$ y el capital relacional $(0.667, \mathrm{p}>0.01)$, siempre y cuando contribuya a la gestión y fortalecimiento del capital intelectual.

\section{Discusión}

Lo apropiado de la aplicación del análisis factorial exploratorio se evidenció al obtener una estructura interna de la escala de capital intelectual, de la cual resultaron cuatro factores (o variables latentes): capital humano, capital estructural, capital relacional y capital tecnológico, que juntos alcanzaron a explicar una varianza total de $66.74 \%$ con 41 reactivos.

El factor con mayor varianza (21.01\%) correspondió al capital estructural integrado de 16 reactivos. Ocho reactivos representan lo estático del capital estructural, y es lo que Brooking (1997) define como conocimiento institucionalizado y experiencia codificada almacenados en bases de datos y patentes, así como manuales, procedimientos, estructuras (niveles jerárquicos y puestos), el sistema de planeación y control de personal y el respectivo de evaluación y promoción, entre otros. En cuanto a lo dinámico del capital estructural, Ramírez, Lorduy, \& Rojas (2007) lo entienden como el conocimiento explícito relacionado con procesos internos de diseminación, comunicación y gestión del conocimiento técnico y científico. Ordoñez (2003) afirma que es el aspecto fundamental para que puedan darse la eficiencia y la eficacia, por lo que a este factor pertenecen ocho reactivos sobre liderazgo, satisfacción laboral, motivación hacia el trabajo, soporte socioemotivo (mejor entendido como clima organizacional) y relaciones humanas. La pertenencia de estos reactivos al capital estructural, y no al capital humano, puede deberse a que como intangibles que generan valor, corresponden más al nivel organizacional que al individual y tratarse de lo que Sánchez et al. (2009) proponen como cultura operativa, toda vez que permite captar la dinámica de la organización.

El capital relacional explicó una varianza de 19.91\% y quedó representado por 13 reactivos sobre el conocimiento que revisten las relaciones entre la organización y sus stakeholders. Incluye la calidad de la relación con instituciones de educación media superior y superior, aunque también con la administración central universitaria.

El factor de capital humano (14.91\% varianza) contiene siete reactivos sobre el nivel educativo, la experiencia, la creatividad, las aptitudes y capacidades, las habilidades para resolver problemas, la obtención de resultados y la actitud hacia el aprendizaje. Para Bezhani (2010), este capital es fuente de innovación y renovación estratégica.

Por último, y sin ser considerado inicialmente como factor de primer grado, el capital tecnológico (10.91\% de varianza explicada) quedó conformado por cinco reactivos referentes a equipo de cómputo (software y hardware), infraestructura para que los académicos realicen su trabajo y se lleve a cabo el proceso enseñanza-aprendizaje, así como la tecnología para educación a distancia.

\section{Conclusiones}

Una fuente de ausencia de información diagnóstica del capital intelectual es la falta de calidad de instrumentos para la recolección de datos primarios y del procedimiento de su aplicación.

Esta escala de medición del capital intelectual se construyó y validó a partir de la teoría de recursos y capacidades, literatura científica y reconociendo el contexto de las universidades mexicanas. Aunque se partió de tres componentes del capital intelectual (capital humano, capital estructural y capital relacional), la escala se validó con cuatro: estos tres y el capital tecnológico.

De los 45 ítems iniciales de la escala de capital intelectual, se eliminaron cuatro $(23,26,29$ y 31$)$ quedando 41, mismos que representan: (a) el capital estructural o conjunto de competencias orga- 
nizativas y estructurales al incorporar, capacitar y sostener el capital humano mediante estructuras, sistemas y procedimientos (16 reactivos); (b) el capital relacional o conjunto de relaciones con el entorno interno y externo (13 reactivos); (c) el capital humano o el conjunto de competencias personales que generan valor para la organización (7 reactivos); y (d) el capital tecnológico o el conjunto de intangibles que permiten la operación dinámica del capital intelectual en las universidades (5 reactivos).

En conclusión, lo aquí presentado es un cuestionario con cierta validez de constructo y se ha avanzado en la consistencia de la escala, ya que permite comprobar que se está midiendo el capital intelectual en el momento en que queda claro que subyacen cuatro factores (capital humano, capital estructural, capital relacional y capital tecnológico), los reactivos que integran cada uno de estos y cómo están relacionados entre sí.

Por ahora, queda en el tintero replicar el estudio en muestras varias para llegar a la certeza de que se trata de una escala multidimensional (y no unidimensional), es decir, que con este cuestionario se están midiendo constructos diferenciados. Lo que sigue es confirmar la trayectoria de los factores del capital intelectual, pues ya se sabe que actúan en interacción.

Las implicaciones prácticas de este estudio radican en que dirigentes, académicos, administrativos, alumnos y todos los agentes vinculados a las universidades sepan que así como "los ingresos visibles incrementan los fondos propios, los ingresos invisibles mejoran el rendimiento y el valor de los activos intangibles" (Sveiby, 1997, p. 302).

Finalmente, mediante la evidencia del capital intelectual, se contribuye a construir argumentos para la asignación de presupuestos y, por ende, su seguimiento y evaluación. Ahora queda a los directivos tener presente que la universidad ostenta el compromiso de contribuir, mediante su liderazgo, sus planes estratégicos y su quehacer cotidiano, a conformar una sociedad más justa en un mundo más plural.

Esta escala es una aportación para medir el carácter dinámico del capital intelectual en universidades, y si bien podría suponerse que cada institución caracteriza su capital intelectual, lo que ya no tiene marcha atrás es que este intangible es esencial en la construcción de ventajas competitivas en la economía del conocimiento.

\section{Referencia}

Aron, A., \&Aron, E. (2001). Estadística para psicología. ( $2^{\mathrm{a}}$ ed.). Buenos Aires: Prentice Hall.

Barney, J. (1991). Firm resources and sustained competitive advantage. Journal of Management, 17(1), 99-120.

Bezhani, I. (2010). Intellectual capital reporting at UK universities. Journal of Intellectual Capital, 11(2), 179207. http://dx.doi.org/10.1108/14691931011039679

Bontis, N. (1998). Intellectual capital: an exploratory study that develops measures and models. Management Decision, 36(2), 63-76. http://dx.doi. org/10.1108/00251749810204142

Brooking, A. (1997). El capital intelectual. España: Paidós Empresa.

Caredda, S. G., D'Egidio, F., \& Gasperini, A. (2004). Medición de intangibles y comunicación en Italia. Valor y aplicaciones del capital intelectual. Economía Industrial, 357, 75-89.

Centro de Investigación sobre la Sociedad del Conocimiento(CIC). (2012). Modelo Intellectus: Medición y Gestión del Capital Intelectual. Documenos Intellectus 9/10. Madrid: CIC/IADE. Recuperado de https://www.academia.edu/7807104/ Modelo_Intellectus

Clark-Carter, D. (2002): Investigación cuantitativa en Psicología. México: Oxford Press.

Duane, D. (2008). Investigación en administración para la toma de decisiones (5 ${ }^{\underline{a}}$ ed.). México: Thomson Editores.

Edvinsson, L., \& Malone, M. (1997). Intellectual capital: Realizing your company's true value by finding its hidden brainpower. Nueva York: Harper Collins Publishers.

Field, A. P. (2003). Designing a questionnaire. Documento de trabajo. Recuperado de http://www.statisticshell.com/docs/designing_questionnaires.pdf

Hair, J., Anderson, R. E., Tatham, R., \& Black, W.C. (1999). Análisis Multivariante (5⿳亠丷厂 ed.). España: Pearson Prentice Hall. 
Huang, Y. C., \& Jim Wu, Y. (2010). Intellectual capital and knowledge productivity: the Taiwan biotech industry. Management Decision, 48(4), 580-599. http://dx.doi.org/10.1108/00251741011041364

Kaplan, R., \& Norton, D. P. (1992).The Balanced Scorecard-Measures that Drive Performance. Harvard Business Review, 70(1), 71-79. Recuperado de http://hbr.org/1992/01/the-balanced-scorecardmeasures-that-drive-performance/ar/1

Kerlinger, F. N., \& Lee, H. B. (2002). Investigación del comportamiento. Métodos de Investigación en Ciencias Sociales (4⿳亠丷a ed.). México: McGraw Hill.

Lev, B., \& Zambon, S. (2003). Intangibles and intellectual capital: an introduction to a special issue. European Accounting Review, 12(4), 597-603. doi: http://dx.doi.org/10.1080/0963818032000162849

Lyn, M. W., \& Bozeman, B. (2006). Researchers' Industry Experience and Productivity in University-Industry Research Centers: A 'Scientific and Technical Human Capital' Explanation. Journal of Technology Transfer, 31, 269-290.

Martínez, M. R., Hernández, M. J., \& Hernández, M. V. (2006). Psicometría. España: Alianza Editorial.

Miles, J., \& Shevlin, M. (2005). Applying regression $\mathbb{E}$ correlation. A guide for students and researchers. London: SAGE.

Morales, P. (2011). El análisis factorial en la construcción e interpretación de tests, escalas y cuestionarios. Madrid: Universidad Pontificia Comillas. Recuperado de http://www.upcomillas.es/personal/peter

Mouritsen, J., Bukh, P. N., \& Marr, B. (2004). Reporting on intellectual capital: why, what and how? Measuring Business Excellence, 8 (1), 46-54. http:// dx.doi.org/10.1108/13683040410524739

Nava, R. M., \& Mercado, P. (2010). Evaluación de la calidad métrica para indicadores de capital intelectual generados a partir de bases estadísticas. Revista de la educación superior, 39(155), 99-120.

Nazari, J., Herremans, I., Manassian, A., \& Kline, T. (2011). Organizational Culture, Climate and IC: An Interaction Analysis. Journal of Intellectual Capital, 12(2), 4-14. http://dx.doi. org/10.1108/14691931111123403

Nunnally, J. C. (2009). Teoría psicométrica. México: Trillas.
Ordoñez, P. (2003). Intellectual capital reporting Spain: A comparative view. Journal of Intellectual Capital, 4(1), 61-81. http://dx.doi. org/10.1108/14691930310455397

Ramírez, Y., Lorduy, C., \& Rojas, J. (2007). Intellectual capital management in Spanish universities. Journal of Intellectual Capital, 8(4), 732-748. http:// dx.doi.org/10.1108/14691930710830873

Roos, J., Roos, G., Dragonetti, N. C., \& Edvinsson, L. (1997). Capital Intelectual. El valor intangible de la empresa. Barcelona: Paidós.

Salmi, J. (2009). El desafío de crear universidades de rango mundial. Colombia: Banco Mundial. Recuperado de http://www.cna.gov.co/1741/articles-311056 UniMundial.pdf

Sánchez, P., Elena, S., \& Castrillo, R. (2009). Intellectual capital dynamics in universities: a reporting model. Journal of Intellectual Capital, 10(2), 307-324. http:// dx.doi.org/10.1108/14691930910952687

Sánchez, R. (2006). Analyzing internal and competitor competences. Resources, capabilities, and management processes. En A. Campbell, \& D. O. Faulkner (Eds.). The Oxford Handbook of Strategy. New York: Oxford University Press.

Stewart, T. (1997). Intellectual Capital. The New Wealth of Organizations. London: Nicholas Brealey Publishing.

Sullivan, P. (2001). Rentabilizar el capital intelectual. Técnicas para optimizar el valor de la innovación. Barcelona: Paidós Ibérica.

Sveiby, K. E. (1997). The new organizational wealth: managing and measuring knowledge-based assets. San Francisco, CA: Berrett-Koehler Publishers.

Tarling, R. (2008). Statistical Modeling for Social Researchers. Principles and practice. Gran Bretaña: Routledge.

Tseng, C., \& James Goo, Y. (2005). Intellectual capital and corporate value in an emerging economy: empirical study of Taiwanese manufacturers. REDD Management, 35(2), 187-201. http://dx.doi. org/10.1111/j.1467-9310.2005.00382.x 


\section{ANEXO}

REACTIVOS DEL CUESTIONARIO DE CAPITAL INTELECTUAL PARA UNIVERSIDADES³

Factor 1. Capital estructural: conocimientos y activos intangibles derivados de los procesos de acción que son propiedad de la organización y que se quedan en ella cuando el personal la abandona.

8. La capacitación que el organismo académico brinda a los empleados

9. El trabajo en equipo

10. El liderazgo

11. La satisfacción del personal

12. La motivación de los empleados

13. El soporte socioemotivo que brinda el organismo académico

14. La relación entre empleados

15. La relación entre directivos y mandos medios

21. Los niveles jerárquicos en el organismo académico

22. Los puestos académico-administrativos

24. El sistema de planeación y control de personal

25. El sistema de evaluación y promoción del personal

27. Los manuales y procedimientos

28. La gestión del conocimiento

30. La cultura organizacional del organismo académico como facilitadora de la productividad y la generación de valor 32. La comunicación de los directivos y profesores con los padres de familia

Factor 2. Capital relacional: conocimiento que se incorpora a la organización y a las personas que la integran como consecuencia del valor derivado de las relaciones continuas con agentes del mercado y la sociedad en general.

33. La comunicación de la institución con los empleados

34. La confianza de los alumnos hacia el organismo académico

35. La confianza de los empleadores hacia la formación de profesionistas

36. La confianza de los egresados hacia el organismo académico

37. La calidad de la relación del organismo académico con oficinas centrales

38. La calidad de la relación del organismo académico con instituciones de educación media superior

39. La calidad de la relación del organismo académico con otras instituciones similares

40. La contribución de este organismo académico a la sociedad mediante la formación de profesionales

41. Las actividades de vinculación del organismo académico con la sociedad

42. La extensión de los servicios del organismo académico

43. La difusión de la cultura en el organismo académico

44. La responsabilidad social del organismo académico

45. La reputación del organismo académico

Factor 3. Capital humano: conocimiento (explícito o tácito e individual o social) que poseen las personas y grupos, así como su capacidad para generarlo, que resulta útil para el propósito estratégico de la organización.

1. El nivel educativo del personal del organismo académico

2. La experiencia del personal del organismo académico

3. La creatividad de los empleados del organismo académico

4. Las aptitudes y capacidades de los empleados del organismo académico

5. La forma de resolver los problemas

6. La obtención de resultados

7. La actitud hacia el aprendizaje

Factor 4. Capital tecnológico: intangibles directamente vinculados con el desarrollo de sistemas técnicos, así como las innovaciones en productos y procesos.

16. La conectividad a Internet

17. El equipo de cómputo (software y hardware) asignado al personal para realizar su trabajo

18. La infraestructura para que los profesores realicen su trabajo

19. La infraestructura para llevar a cabo el proceso enseñanza aprendizaje

20. La infraestructura para la educación a distancia

3 Los reactivos aparecen ordenados por factores según solución factorial final y dentro de cada factor por el número de posición que tienen los reactivos en el cuestionario 
